\title{
Riesgos psicosociales
}

\section{RESUMEN}

El artículo revisa los conceptos de la asignatura pendiente en la prevención de riesgos laborales (PRL): los riesgos psicosociales; constatándose que cada día es más creciente su importancia sobre la salud de los trabajadores y las organizaciones (INSL, Instituto Navarro de Salud laboral, 2005).

El estudio concluye que las leyes de Seguridad y Salud en el Trabajo en el Perú promueven una cultura a la PRL; que se manifiesta en las buenas intenciones de sus artículos. No basta con promover, también es importante la identificación y evaluación en la PRL. Las leyes y reglamentos adolecen de anexos y guías metodológicas de cómo actuar en la identificación y la evaluación de los riesgos psicosociales.

Palabras clave: prevención de riesgos laborales, riesgos psicosociales, accidentes en el trabajo, seguridad y salud ocupacional, sistemas de gestión, violencia laboral, estrés

Psychosocial RISKS

\section{ABSTRACT}

The article reviews the concepts of the unfinished in Occupational Risk Prevention (PRL): Psychosocial risks, thus finding that each day is increasing its importance on the health of workers and organizations (INSL).

The study concludes that the laws of Safety and Health at Work in Peru, to promote a culture PRL, as shown in the good intentions of its articles. That is not enough to promote, it is also important to identify and evaluate the PRL. The laws and regulations and annexes suffer from methodological guidelines on how to act in the identification and assessment of psychosocial risks.

Keywords: prevention of occupational risks, psychosocial risks, accidents at work, safety and occupational health management systems, workplace violence, stress

\section{ANTECEDENTES DE LA INVESTIGACIÓN}

En la evolución de la Seguridad y Salud Ocupacional (SST, SSO o H\&S de Higiene y Seguridad), se menciona desde los simples programas de entrenamiento, pasando por auditorías, realización de análisis de riesgos, hacia técnicas avanzadas como HAZOP y FMEA, Sistemas de Gestión como OHSAS 18000, Sistemas Integrados y desarrollos en Seguridad Basada en Comportamiento como uno de los últimos peldaños de esa gran escalera de la H\&S.

La Seguridad se ocupa de los efectos agudos de los riesgos, es decir, de los accidentes, mientras que la salud ocupacional trata los efectos crónicos de los riesgos, las enfermedades ocupacionales (ver Figura 1).

La Salud Ocupacional es la salud del trabajador en su ambiente de trabajo. Pero, el concepto de salud es mucho más amplio, no solo comprende la salud ocupacional sino también la salud del trabajador fuera de su ámbito laboral. También salud del trabajador, incluye a las patologías asociadas al trabajo y las derivadas de su vida fuera de su centro de labores (ver Figura 2).

En un reciente estudio publicado por The Conference Board, solo la obesidad de los empleados le cuesta U\$ 45,000 millones anuales a las empresas norteamericanas. La razón es sencilla: el ausentismo, generado por las enfermedades ligadas a la obesidad y el incremento en los gastos médicos financiados por las compañías. Aunque la obesidad no sea una enfermedad directamente vinculada con la actividad laboral, el hecho de que genere gastos de tal magnitud hace que se convierta también en un asunto pertinente para la empresa (http://idontec.blogia.com/ temas/la-piramide-de-maslow.php).

La palabra "accidente" tiene su origen en el término latino accidens. El concepto hace referencia a algo que sucede o surge de manera inesperada, ya que no forma parte de lo natural o lo esencial de la cosa en cuestión.

Wikipedia define accidente, a cualquier suceso que es provocado por una acción violenta y repentina ocasionada por un agente externo involuntario, y puede o no dar lugar a una lesión corporal.

* Ingeniero Industrial. Profesor del Departamento Académico de Ingeniería de Sistemas e Informática de la UNMSM. E-mail: eraffolecca@yahoo.es

** Ingeniero Industrial. Profesor del Departamento Académico de Diseño y Tecnología Industrial, de la UNMSM. E-mail: eraezg@unmsm.edu.pe

*** Ingeniero Industrial. Profesor del Departamento Académico de Producción y Gestión Industrial, de la UNMSM. E-mail: orestescachay@yahoo.es 
Figura 1. Seguridad y salud en el trabajo (SST)

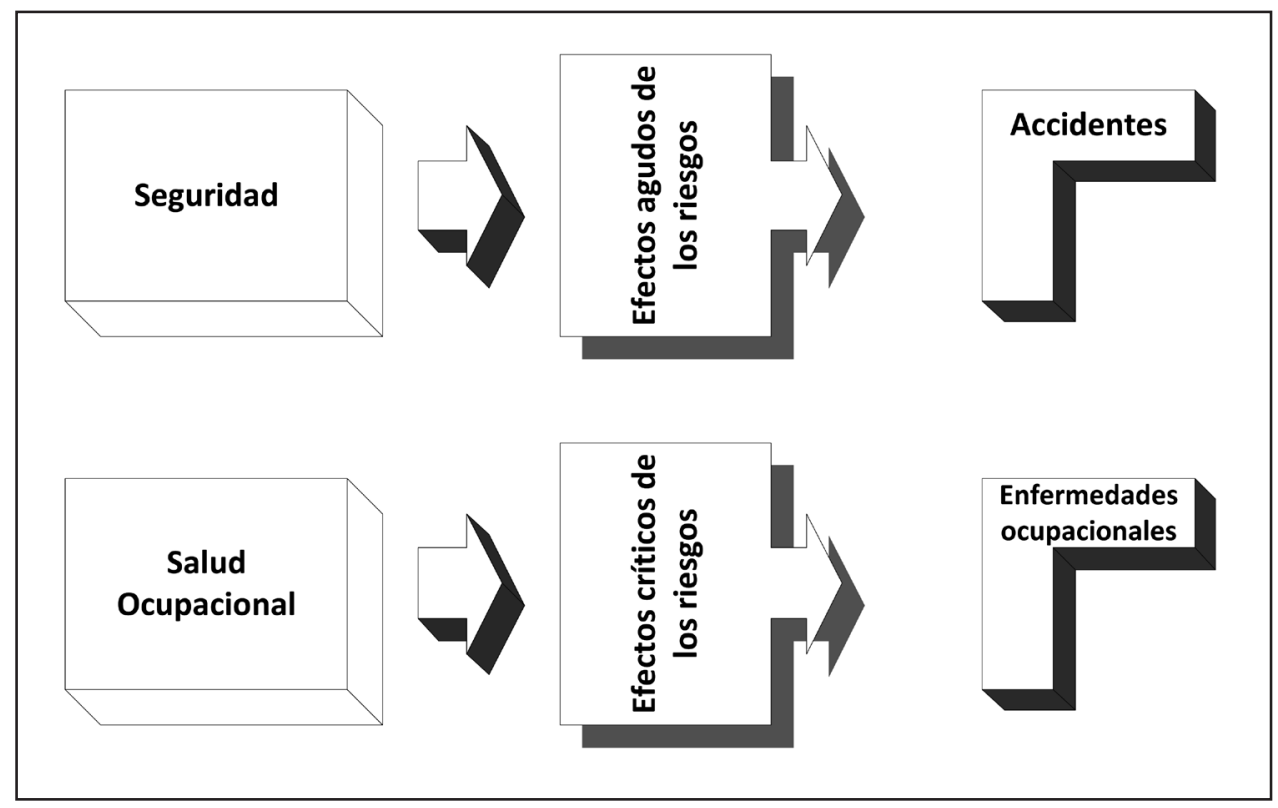

Fuente: Elaboración propia.

La amplitud de los términos de esta definición obliga a tener presente que los diferentes tipos de accidentes se hallan condicionados por múltiples fenómenos de carácter imprevisible e incontrolable (http://es.wikipedia.org/wiki/Accidente).

Según OHSAS 18001:2007 [10], accidente es un incidente que da lugar a lesión, enfermedad o víctima mortal. Incidente es evento relacionado con el trabajo en el que ocurrió o pudo haber ocurrido lesión o enfermedad o víctima mortal.

Según Ramírez Cavassa [12], "Todo accidente es una combinación de riesgo físico y error humano", donde los factores que inciden en la producción del accidente son: humanos y técnicos.

En la Figura 3, se observa que los factores humanos son los sociológicos, económicos, y otros. Los factores técnicos corresponden a la organización. El deterioro o disfunción de los factores da lugar a los accidentes.

La teoría de motivación trata de explicar las condiciones para que los colaboradores puedan trabajar con la menor exposición a los accidentes laborales.

Abraham Maslow, desarrolló la teoría de la motivación, la que se visualiza en la conocida Pirámide de Maslow. Un ser humano tiende a satisfacer sus necesidades primarias (más bajas en la pirámide), antes de buscar las de más alto nivel. Las necesidades fisiológicas (necesidades biológicas básicas de subsistencia, cuya satisfacción es urgente, impostergable más allá de un límite) y con motivadores como el hambre, la sed, el cansancio, el sueño, el apetito sexual) son primarias comparadas con las necesidades de seguridad y estabilidad (como el amor). Luego siguen las necesidades de ego y finalmente las de autorrealización.

Figura 2. Salud del trabajador
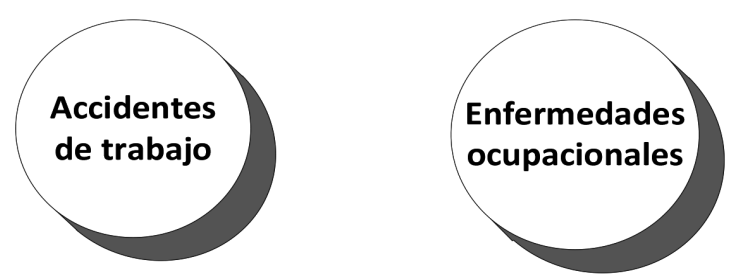

\section{Salud del trabajador}
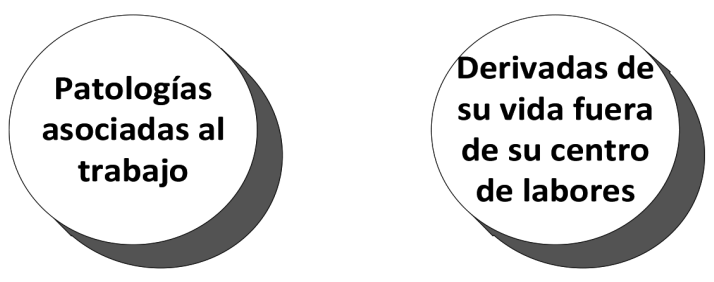

Fuente: Elaboración propia. 
Figura 3. Elementos del accidente.

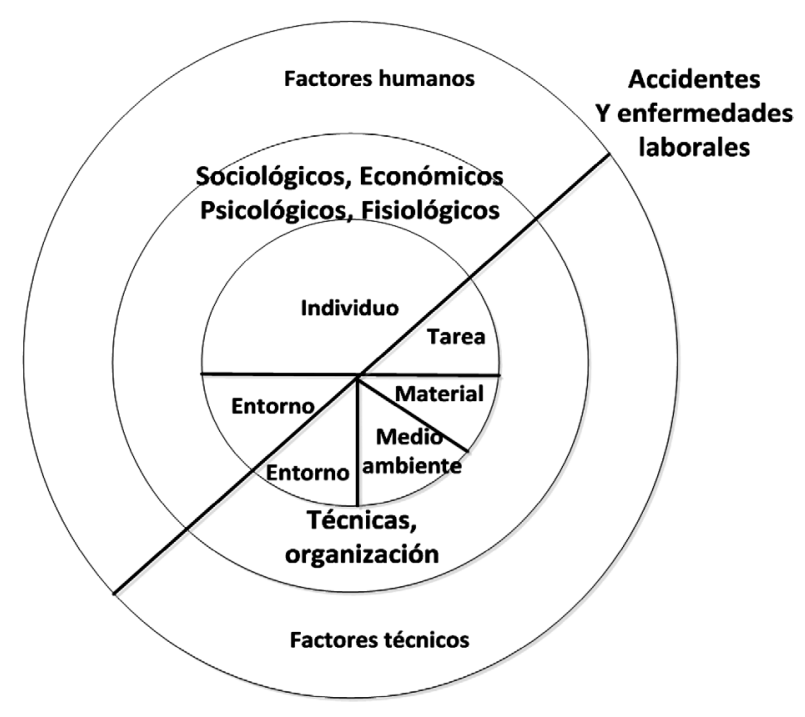

Fuente: Ramírez Cavassa, 2011

Las necesidades de seguridad son las necesidades psicológicas, que dan al individuo tranquilidad y confianza. Entre los motivadores, se encuentran, la certeza sobre algo, el bienestar físico, la salud, el orden. Son los elementos que garantizan la conservación de lo que se ha logrado en el nivel anterior.

Ocurre cuando se le dotan de cascos, guantes, máscaras y otros implementos (EPP), así como la capacitación necesaria para usarlos. Decir no a las condiciones inseguras como los cables de energía protegidos, la señalización correcta de líneas de agua, luz, gas, etc., contribuyen a la seguridad. Es deber del estado, el empresariado y las organizaciones sindicales cumplir el papel de conquistar para sus trabajadores estas condiciones de seguridad

Desde 1969, aparecen los modelos de Sistema de Gestión de Seguridad y Salud Ocupacional (SGSSO o SGSST), pertenecientes a una concepción de problemas multicausal; donde la Alta Dirección es la responsable de establecer, documentar, implementar, mantener y mejorar en forma continua el sistema de gestión. Admitiendo a la auditoría como importante para su avance.

En las directrices relativas a los SGSST de ILO-OSH 2001 (OIT) se destaca:

La protección de los trabajadores contra las enfermedades, dolencias y accidentes relacionados con el trabajo forma parte del mandato histórico de la OIT. La finalidad primordial de la OIT es promover oportunidades para que los hombres y las mujeres puedan conseguir un trabajo decente y productivo en condiciones de libertad, equidad, seguridad y dignidad humana. Esta finalidad se ha resumido en el concepto «trabajo decente». Las organizaciones también deben ser capaces de afrontar los continuos retos de la seguridad y la salud en el trabajo y desarrollar respuestas efectivas en forma de estrategias de gestión dinámicas.

La OIT ha elaborado directrices voluntarias sobre los sistemas de gestión de la SST que reflejan los valores e instrumentos pertinentes de la OIT para la protección de la seguridad y la salud de los trabajadores. Estas directrices forman parte del SafeWork (el programa del trabajo seguro de la OIT).

Implantar un SGSSO, propicia las bases para minimizar los riesgos relevantes a salud, accidentes y otros por seguridad. Esta gestión proporciona un mejor desempeño de las actividades y procesos; resultando en reducción de costos la cual favorece la imagen de la organización ante la comunidad y mercado a la cual la organización provee y beneficios a las utilidades y rentabilidad de la misma.

Muchas empresas desarrollan un Sistema de Gestión de la Salud y Seguridad Ocupacional (SGSSO) como parte de su estrategia de evaluación de riesgos para hacer frente a una legislación cambiante y proteger a su equipo humano. Ver Figura 4.

Figura 4. SGSST.

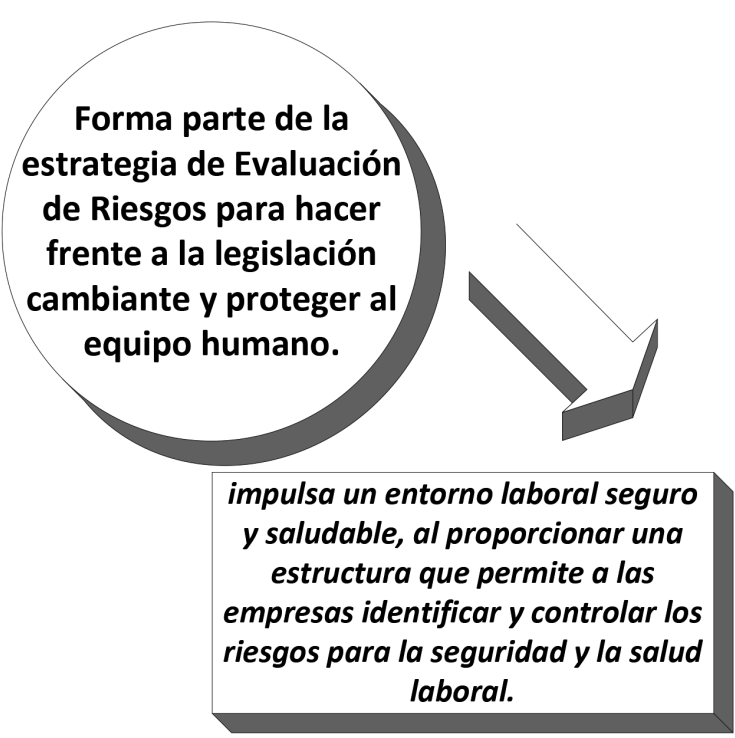

Fuente: Elaboración propia. 
Un SGSST impulsa un entorno laboral seguro y saludable al proporcionar una estructura que permite a las empresas identificar y controlar los riesgos para la seguridad y la salud laborales, reduce la posibilidad de accidentes y ayuda a mejorar el rendimiento general.

OHSAS 18001 (Occupational Health and Safety Assessment Series) es la especificación de evaluación sobre Sistemas de Gestión de Salud y Seguridad Ocupacional, de mayor reconocimiento internacional, desarrollada por un conjunto de importantes organizaciones comerciales y de certificación para cubrir un nicho en lo que a estándares internacionales se refiere.

OHSAS 18001:2007 cubre las siguientes áreas básicas:

- Identificación de amenazas, evaluación de riesgos y establecimiento de controles.

- Requisitos legales y de otro tipo.

- Objetivos y programas.

- Recursos, cargos, responsabilidad, deber y autoridad.

- Competencia, formación y concienciación.

- Comunicación, participación y consultoría.

- Control operacional.

- Preparación y respuesta ante emergencias.

- Medición de la actuación, seguimiento y mejora.

\section{SITUACIÓN PROBLEMÁTICA}

Existe una frase en la sabiduría popular que resume lo que es un principio en materia de seguridad en el trabajo: prevenir antes que lamentar. En efecto, la prevención es un elemento indispensable para alcanzar el principal objetivo de todo sistema de seguridad y salud en el trabajo: evitar pérdidas humanas y materiales en las operaciones.

El trabajo en los momentos actuales, lejos de constituir exclusivamente un medio de subsistencia, constituye un importante elemento de valoración social y de desarrollo de su actividad creadora, constituyéndose en derecho y un deber de la persona (J. Cortés Díaz [2]).

La OIT (Organización Internacional del Trabajo) fue creada en 1919, como parte del Tratado de Versalles que terminó con la Primera Guerra Mundial, y reflejó la convicción de que la justicia social es esencial para alcanzar una paz universal y permanente. En el Preámbulo de la Constitución de la OIT, se encuentra entre otras áreas vigentes, la referida a la protección del trabajador contra enfermedades o accidentes como consecuencia de su trabajo.

Con la Ley de Seguridad y Salud Ocupacionales de 1970, el Congreso USA creó la Administración de Seguridad y Salud Ocupacional (OSHA) a fin de garantizar condiciones de trabajo seguras y salubres para hombres y mujeres trabajadores mediante el establecimiento y la aplicación de normas y el suministro de capacitación, inclusión, instrucción y asistencia. OSHA, es una agencia reguladora y es parte del Departamento de Trabajo. (http://www.osha.gov).

El Instituto Nacional para la Seguridad y Salud Ocupacional (NIOSH) es la agencia federal responsable de hacer investigaciones y recomendaciones para la prevención de las enfermedades y heridas asociadas con el trabajo. $\mathrm{NIOSH}$ es parte del Departamento de Salud y Servicios Humanos (USA).

Según estadísticas de la OIT, OSHA y otras, el indicador de accidentes incapacitantes se ha reducido a casi 1 por cada 1 millón de horas $(0,97$ por millón de horas).

Analizando la pirámide de Frank Bird (Figura 5), y el comportamiento de la accidentalidad de 1, 10, 30, 600 , claramente se refleja que lo más importante es no desperdiciar esfuerzos en los pocos accidentes, sino que se debe concentrar las acciones en la gran base de la pirámide y las grandes oportunidades que existen para mejorar la gestión.

Los factores o riesgos psicosociales, se originan por diferentes aspectos del ambiente y organización del trabajo. Cuando se producen, tienen una incidencia en la salud de las personas a través de mecanismos psicológicos y fisiológicos. La existencia de estos riesgos en el trabajo puede afectar, además de la salud de los trabajadores, al desempeño del trabajo. El estrés (un riesgo moderno en un esquema de trabajo tradicional) y la violencia laboral son los más comunes.

Según NIOSH, el estrés es un problema frecuente y costoso en los lugares de trabajo de la actualidad. Cerca de un tercio de los trabajadores notifican altos niveles de estrés, los cuales están asociados a aumentos significativos en la utilización de los servicios de salud. Adicionalmente, los periodos de discapacidad debido al estrés en el trabajo tienden a ser mucho más prolongados que los de otras lesiones y enfermedades ocupacionales. Como parte integral de una iniciativa de amplio alcance para reducir la incidencia de violencia ocupacional, NIOSH lleva a cabo, financia y publica investigaciones sobre factores de riesgo 
Figura 5. Pirámide de Bird.

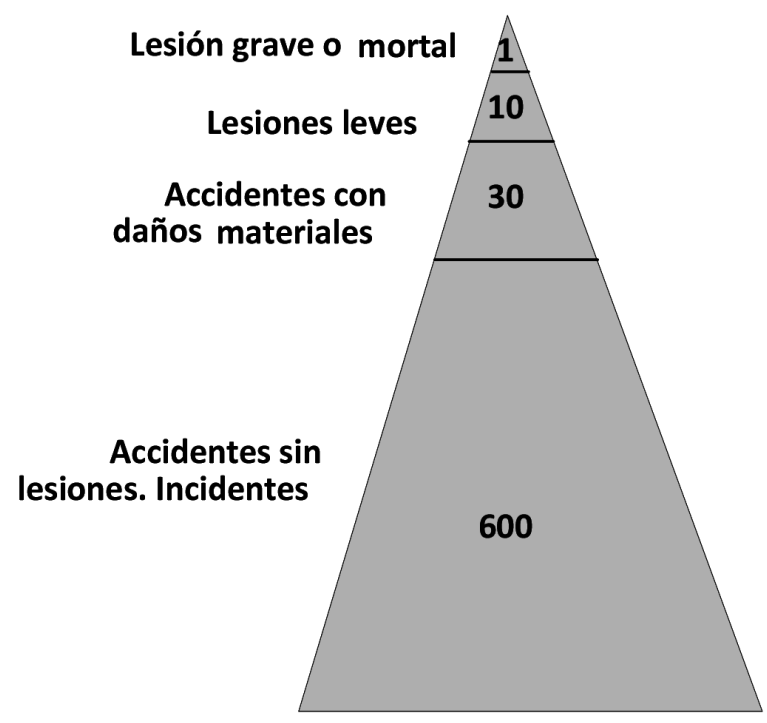

Fuente: Elaboración propia.

y estrategias de prevención (relacionadas con la violencia en el lugar de trabajo (http://www.cdc.gov/ spanish/niosh/topics/default.html).

Según la OIT, en el mundo se registran anualmente cerca de 2 millones de muertes por accidentes o enfermedades laborales, y 160 millones de personas sufren de padecimientos asociados al trabajo. Este impacto económico equivale al 4\% del $\mathrm{PBI}$ del mundo, valor 20 veces superior al de los recursos que se dedican para prevención de riesgos (http://m.elespectador.com).

En el artículo de la OIT, publicado el 5 de julio de 2012 por Seiji Machida Director del Programa Safe Work de la OIT, expresa: "que en la mayoría de los países, el costo total de los accidentes y las enfermedades relativas al ambiente laboral, considerando los asociados con el estrés, es muy alto". En la Unión Europea se estima entre 2,6 y 3,8 por ciento del PIB, donde los estudios sugieren que el estrés es la causa de entre 50 y 60 por ciento de todas las jornadas laborales perdidas.

\section{PREVENCIÓN DE RIESGOS LABORALES}

La prevención exige una evaluación del riesgo en los lugares de trabajo, de manera que se obtenga información precisa para que los diferentes niveles de la organización adopten las medidas preventivas que correspondan. En general, en cada uno de los puestos de trabajo o tareas operativas debe realizarse la evaluación del riesgo, la misma que se caracteriza por ser una actividad dinámica.

En efecto, el cambio significativo en las condiciones de trabajo, la adquisición de nuevas tecnologías operativas, la ocurrencia de accidentes o incidentes recurrentes son algunos indicativos a actualizar la evaluación del riesgo vigente, además de su revisión periódica con participación del personal operativo (Ver Figura 6).

Los procesos de identificación de peligros, evaluación y control de riesgos realizados técnicamente y con la participación del personal que corresponda, permitirán obtener información detallada para establecer las matrices IPER que necesita cada proyecto operativo de la compañía, lo que incluye los controles recomendados de acuerdo a los tipos de peligros y grados de riesgos.

El trabajo, por medio de las modificaciones ambientales o condiciones de trabajo, ejerce sobre la persona una gran influencia; pudiendo llegar a la pérdida del equilibrio de la salud y originar las llamadas patologías del trabajo y sus consecuentes daños derivados del mismo.

Los riesgos psicosociales son una de las áreas en las que se divide tradicionalmente la $\mathrm{PRL}$, siendo las otras áreas la ergonomía, la seguridad y la higiene.

En la Figura 6, se presentan las patologías del trabajo y sus daños derivados. Se observa los tradicionales factores ambientales orgánicos y los nuevos factores que están en el actual ambiente laboral (los psicológicos y sociales), llamados hoy en día riesgos psicosociales.

Figura 6. Prevención de riesgos laborales.

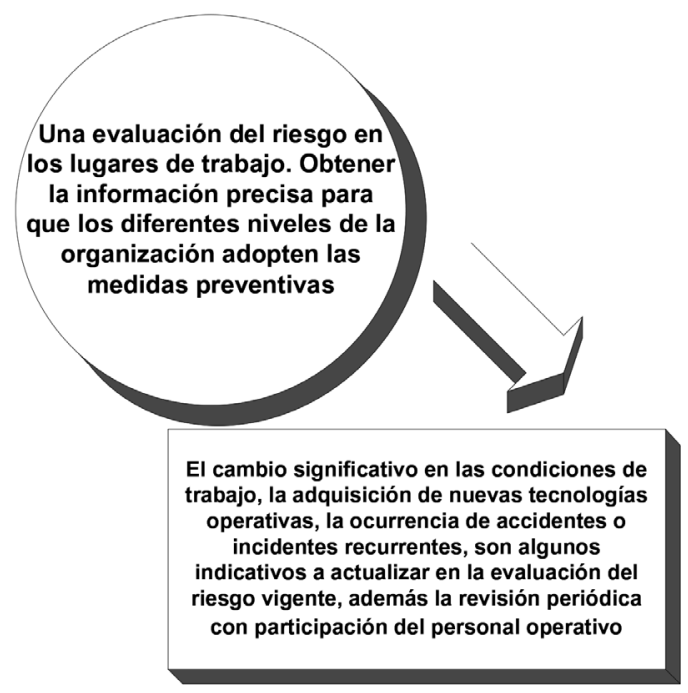

Fuente: Elaboración propia. 


\section{RIESGOS PSICOSOCIALES}

En las Directivas ILO-OSH 2001 aparece la definición de lesiones, dolencias y enfermedades relacionadas con el trabajo:

Efectos negativos en la salud de una exposición en el trabajo a factores químicos, biológicos, físicos, psicosociales o relativos a la organización del trabajo.

En el manual de Riesgos Psicosociales en el mundo laboral de la UGT Aragón (España), se indica que el estudio de los aspectos organizacionales y psicosociales y su relación con la salud laboral, no es nuevo aunque si la importancia y reconocimiento que ha adquirido en los últimos años (Agencia Europea para la SST o EU-OSHA, 2002).

Agrega que: como consecuencia de los importantes cambios en las organizaciones y de los procesos de globalización actual, la exposición a los riesgos psicosociales se ha hecho más frecuente e intensa, haciendo conveniente y necesario su identificación, evaluación y control con el fin de evitar sus riesgos asociados para la salud y la seguridad en el trabajo (EU-OSHA, 2007).
En el informe de la OIT (2005) titulado Trabajo decente-Trabajo seguro, presentado al XVII Congreso Mundial sobre Salud y Seguridad en el Trabajo, Orlando, Estados Unidos, se indica en los problemas emergentes como los relativos a factores psicosociales, casos de violencia, los efectos del alcohol y las drogas, el estrés, el consumo de tabaco y el $\mathrm{VIH} /$ Sida dan lugar a un rápido incremento de la morbilidad y la mortalidad en todo el mundo (http:// www.ilo.org/global/about-the-ilo/newsroom/news/ WCMS_006117/lang--es/index.htm)

La OIT tiene el programa educativo denominado Abordar los problemas psicosociales en el trabajo (SOLVE), que actúa en numerosos países para abordar tales problemas, tanto en el ámbito de la formulación de políticas, como en los centros de trabajo.

El Informe del Comité Mixto OIT-OMS sobre medicina del trabajo, novena reunión Ginebra, 18-24 de septiembre de 1984, plantea la definición de factores psicosociales:

Los factores psicosociales en el trabajo consisten en interacciones entre el trabajo, su medio

Figura 7. Patologías del trabajo.

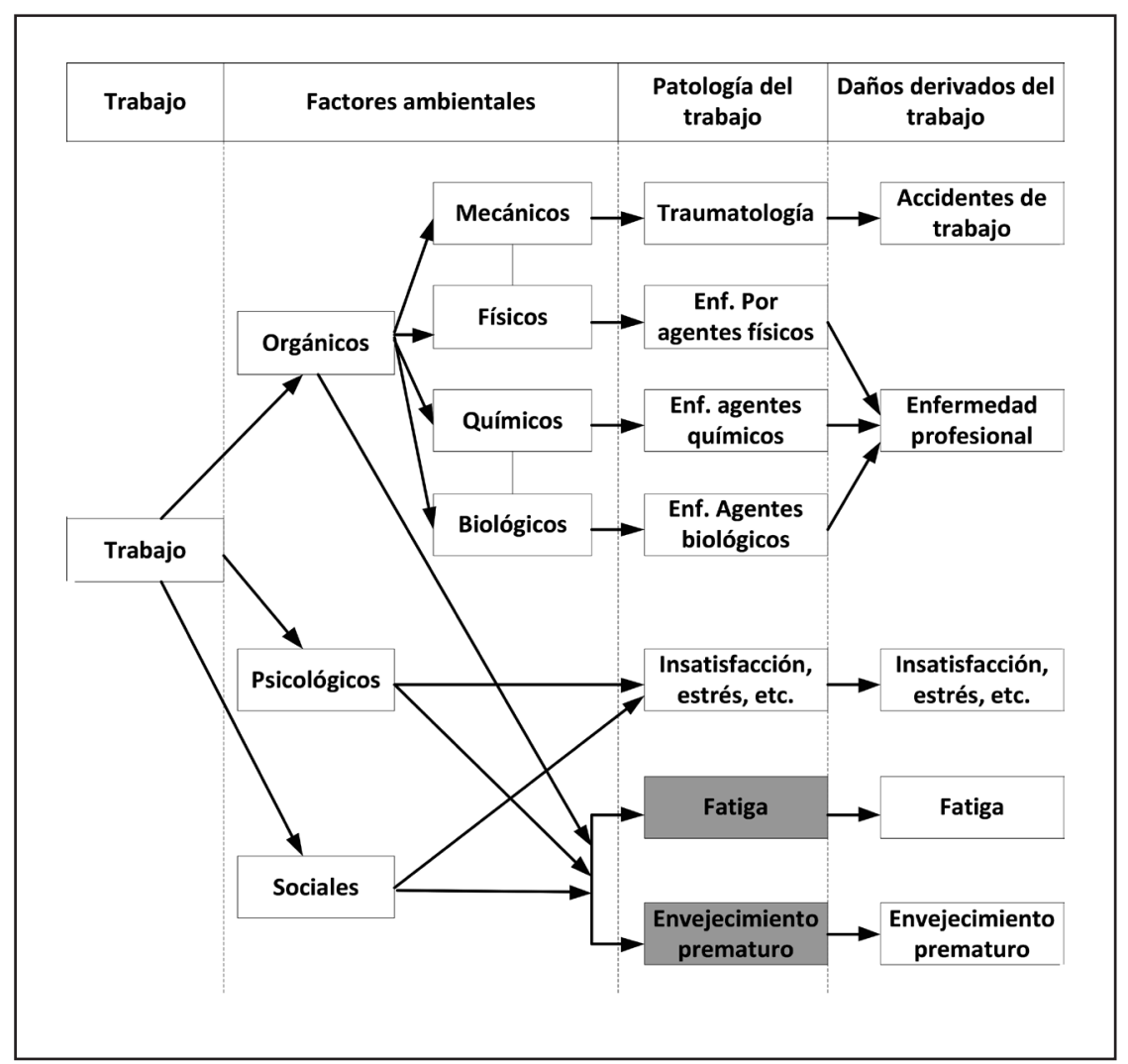

Fuente: J. Cortés Díaz, 2012 
ambiente, la satisfacción en el trabajo y las condiciones de su organización, por una parte, y por la otra, las capacidades del trabajador, sus necesidades, su cultura y su situación personal fuera del trabajo, todo lo cual, a través de percepciones y experiencias, pueden influir en la salud y en el rendimiento y la satisfacción en el trabajo.

La Figura 8 presenta el conjunto de interacciones del medio ambiente de trabajo que están en continua interacción dinámica. El medio ambiente de trabajo, las relaciones entre los trabajadores, las tareas y los factores de organización son representativos de las condiciones de trabajo.

Elinformecontinuacon: "Lainteracciónnegativaentre las condiciones de trabajo y los factores humanos del trabajador pueden conducir a perturbaciones emocionales, problemas del comportamiento y cambios bioquímicos y neurohormonales que presentan riesgos adicionales de enfermedades mentales y físicas".

El informe del comité mixto OIT-OMS a partir de numerosos estudios realizados, identifica en el medio ambiente de trabajo, una serie de factores psicosociales, potencialmente negativos, vinculados con la salud. Tales factores son: la mala utilización de habilidades, la sobrecarga de trabajo, la falta de control, el conflicto de autoridad, la desigualdad en el salario, la falta de seguridad en el trabajo, los problemas en las relaciones laborales, el trabajo por turnos y el peligro físico.

Agrega el informe usando la terminología de Cortez [2]: "Los estudios confirman que la salud física peligra cuando el medio de trabajo deshumaniza a la persona por una combinación de factores que exponen a riesgos físicos y mentales".

Los factores orgánicos, con la utilización de instrumentos y métricas adecuadas son identificados y luego evaluados. Un sonómetro mide el ruido de una fuente a través de los decibeles y es comparado con el límite permisible. La problemática es cómo identificar los riesgos psicosociales y su posterior evaluación.

El Observatorio Permanente de Riesgos Psicosociales de la UGT a partir de las indicaciones de organismos como OIT y OMS, para los riesgos "emergentes "relacionados con la actividad laboral; considera que los riesgos psicosociales visto como una unidad, engloban una variedad de situaciones. El caso más típico es el conocido estrés laboral o estrés ocupacional y su conexo el agotamiento o

Figura 8. Interacciones del medio ambiente de trabajo.

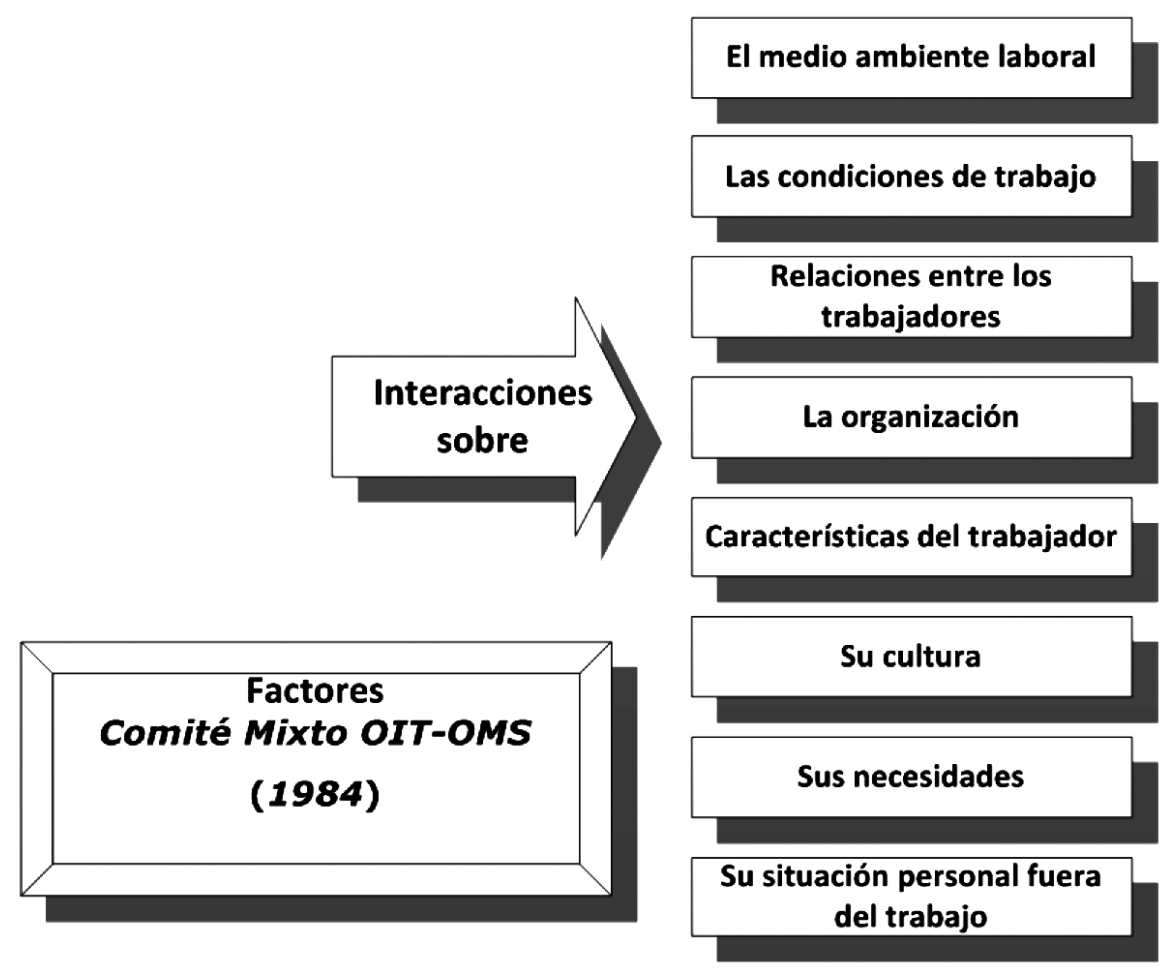

Fuente: Elaboración propia. 
desgaste profesional conocido "síndrome de estar quemado por el trabajo" o Burnout. Sus efectos son psicológicos, psicosomáticos y psicosociales. (Ver Figura 9).

Según el INSHT (2010) se considera también riesgos psicosociales a la violencia laboral, el acoso sexual, el acoso moral y la inseguridad contractual. Al acoso moral o psicológico también se le denomina mobbing (los anglosajones usan el término bullying).

En casos extremos, el estrés puede conducir al suicidio. Seiji Machida director del programa Safe Work de la OIT (2012) advierte sobre un aumento general de las enfermedades mentales debido a la tensión en el ambiente laboral. Mencionan entre otras causas: "exceso de información, intensificación del trabajo y de las presiones temporales, mayores exigencias de movilidad y flexibilidad, estar siempre disponible debido a la tecnología de los teléfonos móviles y, por último pero no menos importante, el miedo de perder el trabajo".

De todo lo expuesto se tienen las siguientes relaciones: las condiciones psicosociales tienen su efecto en la salud mental y emocional. Estas a su vez están relacionadas con la seguridad, e inciden en la productividad, calidad y sostenibilidad de la organización.

\section{RIESGOS PSICOSOCIALES EN LA LEGISLA- CIÓN PERUANA}

El artículo 7 de la Constitución Política, reconoce el derecho de la salud de toda persona en cualquier ámbito, incluido el laboral.

La seguridad y la salud en el trabajo, es una condición básica para la protección social y el desarrollo de las relaciones de trabajo decentes.

Figura 9. Efectos de los factores.

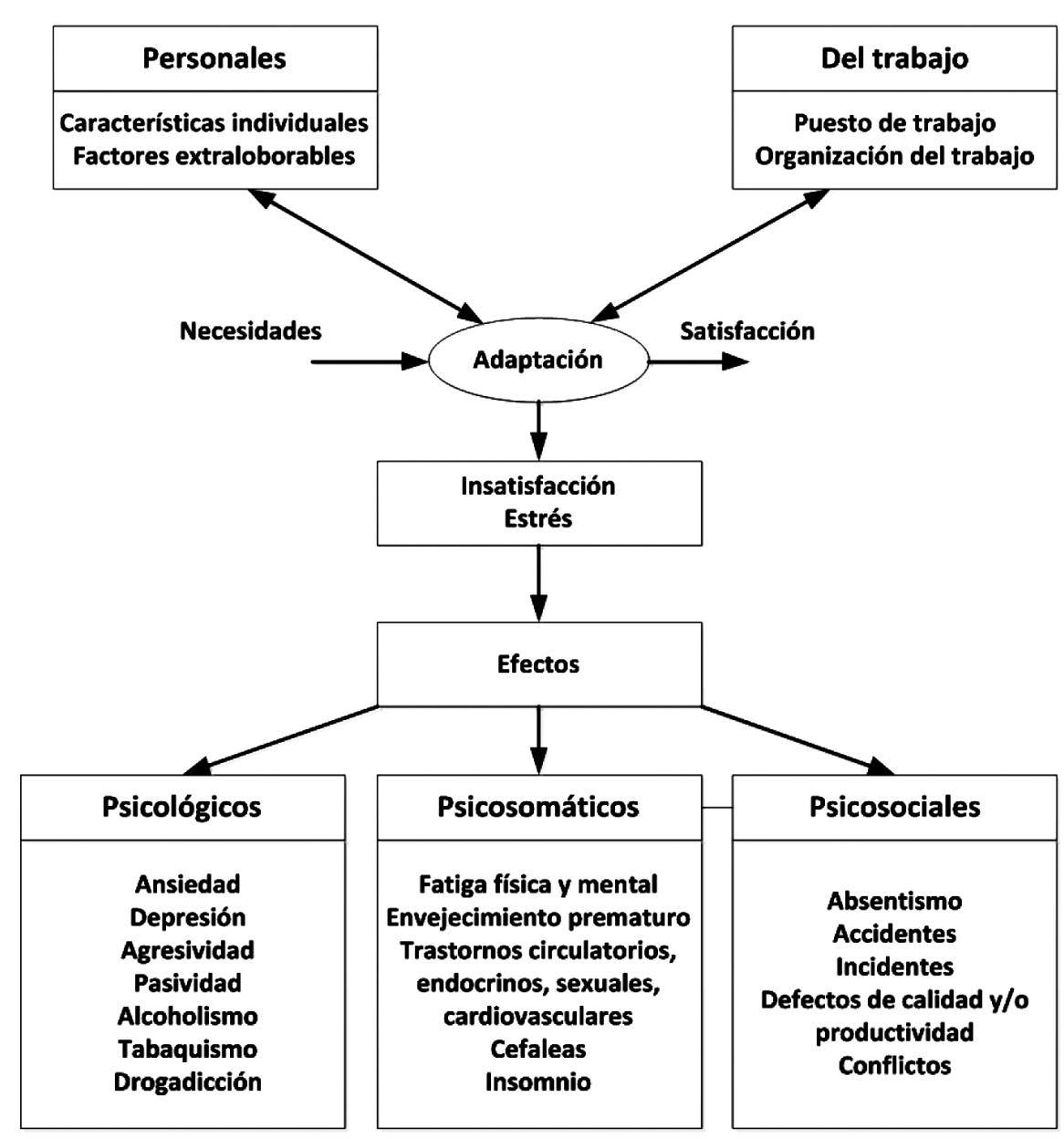

Fuente: Elaboración propia. 
El reglamento DS-055-2010-EM reemplaza al ReglamentodeSeguridadeHigieneMinera aprobado mediante Decreto Supremo N. ${ }^{\circ}$ 046-2001-EM y sus modificatorias; quedando sin efecto a la entrada en vigencia del Reglamento de Seguridad y Salud Ocupacional.

En el artículo 1, se declara:

El presente reglamento tiene como objetivo prevenir la ocurrencia de incidentes, accidentes y enfermedades ocupacionales, promoviendo una cultura de prevención de riesgos laborales en la actividad minera Para ello cuenta con la participación de los trabajadores, empleadores y el Estado, quienes velarán por su promoción, difusión y cumplimiento.

La Ley de Seguridad y Salud en el Trabajo 29783 (2011), en el Principio I: Principio de Prevención, dice: que el empleador garantiza, en el centro de trabajo, el establecimiento de los medios y condiciones que protejan la vida, la salud y el bienestar de los trabajadores. Por otro lado, el empleador debe considerar factores sociales, laborales y biológicos.

En el Principio IX: Principio de Protección, dice: los trabajadores tienen derecho a que el Estado y los empleadores aseguren condiciones de trabajo dignas que les garanticen un estado de vida saludable, física, mental y socialmente, en forma continua.

El artículo 1, Objeto de la Ley declara:

La Ley de SST tiene como objetivo promover una cultura de prevención de riesgos laborales en el país. Para ello, cuenta con el deber de prevención de los empleadores, el rol de fiscalización y control del Estado y la participación de los trabajadores y sus organizaciones sindicales.

El artículo 56, Exposición en zonas de riesgo declara:

El empleador prevé que la exposición a los agentes físicos, químicos, biológicos, ergonómicos y psicosociales concurrentes en el centro de trabajo no generen daños a la salud de los trabajadores.

El artículo 65, Evaluación de factores de riesgo para procreación declara:

En las evaluaciones del plan integral de prevención de riesgos, se tiene en cuenta los factores de riesgos que pueden incidir en las funciones de procreación de los trabajadores, en particular, por la exposición a los agentes físicos, químicos, biológicos, ergonómicos y psicosociales, con el fin de adoptar las medidas preventivas asociadas.

El Reglamento de la Ley N. ${ }^{\circ} 29783$, DS N. ${ }^{\circ}$ 005-2012-TR en el artículo 103 declara lo siguiente:
De conformidad con el artículo 56 de la Ley, se considera que existe exposición a los riesgos psicosociales cuando se perjudica la salud de los trabajadores, causando estrés y, a largo plazo, una serie de sintomatologías.

El artículo $5^{\circ}$ de la Ley N. $^{\circ} 27711$, Ley del MTPE, señala que el Sector Trabajo tiene como atribuciones definir, concertar, coordinar, dirigir, supervisar y evaluar la política de higiene y seguridad ocupacional, y establecer las normas de prevención y protección contra riesgos ocupacionales que aseguren la salud integral de los trabajadores, en aras del mejoramiento de las condiciones y el medio ambiente de trabajo.

Que, mediante la Ley N. ${ }^{\circ} 28048$, Ley de protección a favor de la mujer gestante que realiza labores que pongan en riesgo su salud y/o el desarrollo normal del embrión y el feto, se faculta a las mujeres gestantes para que en sus centros de trabajo soliciten al empleador no realizar labores que pongan en peligro su salud y/o la del desarrollo normal del embrión y el feto durante el periodo de gestación.

Teniendo en cuenta las disposiciones contenidas en la Ley N. ${ }^{\circ} 28048$ y sus Normas Reglamentarias, se aprueba el "Listado de los agentes físicos, químicos, biológicos, ergonómicos y psicosociales que generan riesgos para la salud de la mujer gestante y/o el desarrollo normal del embrión y el feto". Donde se incluyen ocho (08) anexos, siendo el anexo 4 el Listado de Agentes Psicosociales.

\section{CONCLUSIONES}

1. Los factores psicosociales están identificados, como también los riesgos psicosociales por toda la comunidad en Prevención de Riesgos Laborales o PRL.

2. Las leyes de SST en el Perú, promueven una cultura a la PRL; que se muestra en las buenas intenciones de sus artículos. No basta con promover, también es importante la identificación y evaluación en la PRL. Las leyes y reglamentos adolecen de anexos y guías metodológicas de cómo actuar en la identificación y la evaluación de los riesgos psicosociales.

3. Se hace imprescindible de una guía de PRL en riesgos psicológicos para su uso en las organizaciones en nuestro país. Esta labor puede ser originada por el sector académico, el sector empresariado o del Estado. 


\section{REFERENCIAS BIBLIOGRÁFICAS}

[1] Accidente, Wikipedia. http://es.wikipedia.org/ wiki/Accidente, Visitado el 6 de enero 2013.

[2] Cortez Díaz, J. (2012). Técnicas de prevención de riesgos laborales: Seguridad e Higiene en el trabajo. Madrid, España: Tebar, S.L.

[3] El espectador. http://m.elespectador.com/ impreso/negocios/articuloimpreso-invertirsalud-y-seguridad, visitado el 6 de enero 2013.

[4] Comité Mixto OIT-OMS (1984). Factores Psicosociales en el Trabajo. OIT-Ginebra.

[5] INSHT (2010). Factores y riesgos psicosociales, formas, consecuencias, medidas y buenas prácticas. Madrid, España.

[6] ILO-OSH 2001. Directrices relativas a los Sistemas de Gestión y Salud en el Trabajo. OITGinebra.
[7] NIOSH. http://www.cdc.gov/spanish/niosh/ topics/default.html, Visitado el 6 de enero 2013.

[8] OIT, Trabajo decente - Trabajo seguro (2005). Congreso Mundial sobre Salud y Seguridad en el Trabajo, Orlando, Estados Unidos. http:// www.ilo.org/global/about-the-ilo/newsroom/ news/WCMS_006117/lang--es/index.htm, visitado el 6 de Enero 2013.

[9] Observatorio Permanente Riesgos Psicosociales - UGT (2006). Guía sobre el estrés ocupacional. www.ugt.es

[10] OSHA. http://www.osha.gov/about-sp.html, visitado el 6 de enero 2013.

[11] The Conference Board. http://idontec.blogia. com/temas/la-piramide-de-maslow.php, visitado el 6 de enero 2013.

[12] Ramírez Cavassa, C. (2011). Seguridad: un enfoque Integral". México: Editorial Limusa, S.A. de C.V., Grupo Noriega Editores. 